NYU-TH/99/2/02

September 3, 2018

\title{
On Field/String Theory Approach to Theta Dependence in Large $N$ Yang-Mills Theory
}

\author{
Gregory Gabadadze \\ Department of Physics, New York University, New York, NY 10003, USA \\ email: gabadadze@physics.nyu.edu
}

\begin{abstract}
The theta dependence of the vacuum energy in large $N$ Yang-Mills theory has been studied some time ago by Witten using a duality of large $N$ gauge theories with string theory compactified on a certain space-time. We show that within the field theory context vacuum fluctuations of the topological charge give rise to the vacuum energy consistent with the string theory computation. Furthermore, we calculate $1 / N$ suppressed corrections to the string theory result. The reconciliation of the string and field theory approaches is based on the fact that the gauge theory instantons carry zerobrane charge in the corresponding $D$-brane construction of Yang-Mills theory. Given the formula for the vacuum energy we study certain aspects of stability of the false vacua of the model for different realizations of the initial conditions. The vacuum structure appears to be different depending on whether $N$ is infinite or, alternatively, large but finite.
\end{abstract}




\section{Introduction}

A great deal of information can be learned on nonperturbative phenomena in fourdimensional gauge theories by obtaining these models as a low-energy realization of certain D-brane configurations [1], and/or using a duality of large $N$ superconformal gauge theories and string theory compactified on certain spaces (see Refs. [2] and [3, [4]). This duality, being a powerful technique, has also been generalized for the case of non-supersymmetric models [5]. This was applied to study various dynamical issues in large $N$ pure Yang-Mills theory [6] [1]. In fact, it was used by Witten [12] to derive the expression for the theta dependence of the vacuum energy in the large $N$ pure Yang-Mills (YM) model. The expression for the vacuum energy density associated with the $\mathrm{CP}$ odd part of the theory has the form [12]

$$
\mathcal{E}_{0}(\theta)=C \min _{k}(\theta+2 \pi k)^{2}+\mathcal{O}\left(\frac{1}{N}\right)
$$

where $C$ is some constant independent of $N$ and $k$ stands for an integer number. This expression has a number of interesting features which might seem a bit puzzling from the field theory point of view. Indeed, in the large $N$ limit there are $N^{2}$ degrees of freedom in gluodynamics, thus, naively, one would expect that the vacuum energy density in this theory scales as $\sim N^{2}$. However, the leading term in Eq. (西) scales as $\sim 1$. As a natural explanation, one could conjecture that there should be a colorless massless excitation which saturates the expression for the vacuum energy density (11). However, pure gluodynamics generates a mass gap and there are no physical massless excitations in the model. Thus, the origin of Eq. (1) seems to be a conundrum.

In this work we intend to elucidate the field-theoretic origin of Eq. (1). In fact, we identify a very special composite field which defines the vacuum energy given in (1). Moreover, we show that this composite field does not propagate physical degrees of freedom. Thus, we clarify the puzzle mentioned above: The composite field sets the background energy density but does not appear in the spectrum as a physical state. Using this field we calculate $1 / N$ suppressed corrections to (11).

In section 2, after a brief discussion of the theta dependence in pure gluodynamics we derive the expression (11) within the field theory context. Then, we elucidate why the composite field defining the background energy (1) does not propagate dynamical degrees of freedom. In section 3 the same derivation will be given by starting with QCD with massive quarks and recovering pure gluodynamics in the limit when the quark masses go to infinity. Doing so we establish the limits of applicability of the results of section 2 and, most importantly, we calculate the $1 / N$ corrections to the vacuum energy density (1). In section 4 we discuss the fate of the false vacua emerging in the theory as a result of the energy dependence given in (11). We study this issue for both, the large but finite $N$ and infinite $N$ cases. Different realizations of the initial conditions for the model will be considered. In section 5 we discuss the origin of the vacuum energy as it appears in the string theory and field theory approaches. In fact, we analyze certain relations between these two calculations using the arguments of the AdS/CFT correspondence [1]. Section 6 is devoted to brief

*It has been conjectured long time ago in [13]. 
discussions and conclusions.

\section{Derivation in gluodynamics}

The aim of this section is to derive Eq. (11) in pure YM model and, in fact, to identify the degrees of freedom which are responsible for the theta dependent vacuum energy density.

In the quasi-classical approach the theta dependence can be calculated using instantons [14. In a simplest approximation of non-interacting instantons the theta angle enters the Euclidean space partition function in the following form:

$$
\exp \left(-\frac{8 \pi^{2}}{g^{2}} \pm i \theta\right) \equiv \exp \left(-N \frac{8 \pi^{2}}{\lambda} \pm i \theta\right)
$$

where $g$ stands for the strong coupling constant. $\lambda$ denotes the 't Hooft's coupling $\lambda \equiv N g^{2}$ which is kept fixed in the large $N$ limit. The expression above vanishes in the large $N$ limit, so does the theta dependence in (2). However, this conclusion cannot be extrapolated to the infrared region of the model. The limitations of the expression (2) prevent one to do so. Indeed, the quasi-classical approximation is valid in the limit of small coupling constant (see, for instance, discussions in Ref. [15]). Once quantum corrections are taken into account the coupling constant $g^{2}$ in (2) becomes a scale dependent quantity. In fact, it will depend upon an instanton size $g^{2}=g^{2}(\rho)$. For small size instantons the running coupling is small and the quasi-classical approximation in (2) holds. However, for large size instantons, i.e. large couplings, it is not even clear whether the notion of a single instanton is a legitimate approximation. The overlap between instantons can be big in this case and some more complicated field configurations should be relevant for the description of physical phenomena [16]. In any event, the expression (2) is no longer reliable in the strong coupling limit. Thus, the conclusion that the theta dependence goes away in the large $N$ limit cannot be justified. One way to study the infrared region is to look for some appropriate composite colorless excitations for which the notion of an asymptotic state can be used. We will start by searching for these excitations in pure Yang-Mills theory. To proceed, let us recall that the topological susceptibility, $\mathcal{X}$, is a nonzero number in pure gluodynamics:

$$
\mathcal{X}=-i \int \partial^{\mu} \partial^{\nu}\left\langle 0\left|T K_{\mu}(x) K_{\nu}(0)\right| 0\right\rangle d^{4} x \neq 0
$$

Here, $K_{\mu}$ denotes the Chern-Simons current which is related to the topological charge density as $Q=\partial^{\mu} K_{\mu}=\frac{g^{2}}{32 \pi^{2}} F_{\mu \nu} \tilde{F}^{\mu \nu} \equiv F \tilde{F}$, and the dual strength-tensor is defined as $\tilde{F}^{\mu \nu}=$ $\frac{1}{2} \varepsilon^{\mu \nu \alpha \beta} F_{\alpha \beta}$. All quantities above are defined in Minkowski space币. The value of $\mathcal{X}$ in large $N$ pure YM theory determines the $\eta^{\prime}$ meson mass in full QCD with massless quarks. This relation, called the Witten-Veneziano formula, can be written as $m_{\eta^{\prime}}^{2} f_{\eta^{\prime}}^{2} \propto \mathcal{X}$, with $f_{\eta^{\prime}}$ being

\footnotetext{
${ }^{\dagger}$ Though in Minkowski space-time $Q$ does not have the meaning of the topological charge density and, moreover, differs from the Euclidean definition of the topological charge density by $i$, we formally keep the same name and letter for simplicity.
} 
the $\eta^{\prime}$ meson decay constant [17,18].

In what follows it will prove convenient to introduce a new variable by rewriting the expression for the topological charge density $Q$ in terms of a four-index (four-form) tensor field $H^{\mu \nu \alpha \beta}$ :

$$
F \tilde{F}=Q=\frac{\varepsilon_{\mu \nu \alpha \beta} H^{\mu \nu \alpha \beta}}{4 !}
$$

where the four-form field $H^{\mu \nu \alpha \beta}$ is the field strength for the three-form potential $C_{\mu \nu \alpha}$ :

$$
H_{\mu \nu \alpha \beta}=\partial_{\mu} C_{\nu \alpha \beta}-\partial_{\nu} C_{\mu \alpha \beta}-\partial_{\alpha} C_{\nu \mu \beta}-\partial_{\beta} C_{\nu \alpha \mu} .
$$

The $C_{\mu \nu \alpha}$ field is defined as a composite operator of the gluon fields $A_{\mu}^{a}$ :

$$
C_{\mu \nu \alpha}=\frac{1}{16 \pi^{2}}\left(A_{\mu}^{a} \bar{\partial}_{\nu} A_{\alpha}^{a}-A_{\nu}^{a} \bar{\partial}_{\mu} A_{\alpha}^{a}-A_{\alpha}^{a} \bar{\partial}_{\nu} A_{\mu}^{a}+2 f_{a b c} A_{\mu}^{a} A_{\nu}^{b} A_{\alpha}^{c}\right) .
$$

Here, $f_{a b c}$ denote the structure constants of the corresponding $S U(N)$ gauge group. The right-left derivative in this expression is defined as $A \bar{\partial} B \equiv A(\partial B)-(\partial A) B$. Notice, that the $C_{\nu \alpha \beta}$ field is not a gauge invariant quantity; if the gauge transformation parameter is $\Lambda^{a}$, the three-form field transforms as $C_{\nu \alpha \beta} \rightarrow C_{\nu \alpha \beta}+\partial_{\nu} \Lambda_{\alpha \beta}-\partial_{\alpha} \Lambda_{\nu \beta}-\partial_{\beta} \Lambda_{\alpha \nu}$, where $\Lambda_{\alpha \beta} \propto A_{\alpha}^{a} \partial_{\beta} \Lambda^{a}-A_{\beta}^{a} \partial_{\alpha} \Lambda^{a}$. However, one can check that the expression for the field strength $H_{\mu \nu \alpha \beta}$ is gauge invariant.

It has been known for some time [22] that the $C_{\nu \alpha \beta}$ field propagates long-range correlations if the topological susceptibility is nonzero in the theory. The easiest way to see this is to turn to the notion of the Kogut-Susskind pole [23]. Let us consider the correlator of the vacuum topological susceptibility at a nonzero momentum. In this case $\mathcal{X}$ is defined as the zero momentum limit of the correlator of two Chern-Simons currents multiplied by two momenta:

$$
\mathcal{X}=-i \lim _{q \rightarrow 0} q^{\mu} q^{\nu} \int e^{i q x}\left\langle 0\left|T K_{\mu}(x) K_{\nu}(0)\right| 0\right\rangle d^{4} x
$$

Since this expression is nonzero, it must be that the correlator of two Chern-Simons currents develops a pole as the momentum vanishes, the Kogut-Susskind pole [23].

Given that the correlator of two Chern-Simons currents has a pole and that the ChernSimons current and the three-form $C_{\nu \alpha \beta}$ field are related, one concludes that the $C_{\nu \alpha \beta}$ field also has a nonzero Coulomb propagator [22]. Thus, the $C_{\nu \alpha \beta}$ field behaves as a massless collective excitation propagating a long-range interaction [22]. These properties, in the large $N$ limit, can be summarized in the following effective action for the $C_{\nu \alpha \beta}$ field:

$$
S_{e f f}=-\frac{1}{2 \cdot 4 ! \mathcal{X}} \int H_{\mu \nu \alpha \beta}^{2} d^{4} x-\frac{\theta}{3 !} \int_{\partial \Gamma} C_{\nu \alpha \beta} d x^{\nu} \wedge d x^{\alpha} \wedge d x^{\beta}+\text { High dim. }
$$

\footnotetext{
$\ddagger$ The numerical value for $\mathcal{X}$ can also be found within the framework of QCD sum rule [19] calculations [20], as well as in lattice studies [21]. Below, unless otherwise stated, we will not distinguish between $\mathcal{X}$ and its large $N$ limit. The constant contact term in the definition of $\mathcal{X}$ will also be omitted for simplicity.
} 
The first term in this expression yields the correct Coulomb propagator for the three-form $C_{\nu \alpha \beta}$ field. The second term is just the usual CP odd $\theta$ term of the initial YM action written as a surface integral at spatial infinity $\partial \Gamma$. Notice that higher dimensional terms are not explicitly written in this expression. There might be two types of higher dimensional contributions in (8). First of all, there are terms with higher powers of derivatives of the fields. These terms are suppressed by momenta of the "massless" three-form field and do not contribute to the zero momentum vacuum energy of the system. In addition, there might be higher dimensional terms with no additional derivatives. In the next section we will present some of these contributions and show that they are suppressed by higher powers of $1 / N$.

In what follows we are going to study the large $N$ effective action given in Eq. (8) 3 . In particular, we will calculate the ground state energy of the system in the large $N$ limit using the effective action (8). In fact, we will derive Eq. (1).

Before we turn to this calculation let us mention that Maxwell's equations for a free fourform field-strength $H_{\mu \nu \alpha \beta}$ yield only a constant solution in $(3+1)$-dimensional space-time [27]. The reason is as follows. A four-form potential has only one independent degree of freedom in four-dimensional space-time, let us call it $\Sigma$. Then, the four Maxwell's equations written in terms of the $\Sigma$ field require that this field is independent of the all four space-time coordinates, thus the solution can only be a space-time constant. As a result, the free $H_{\mu \nu \alpha \beta}$ field propagates no dynamical degrees of freedom in $(3+1)$-dimensions. However, this field can be responsible for a positive vacuum energy density in various models of Quantum Field Theory (see Ref. [28]). Thus, studying classical equations of motion for the $H_{\mu \nu \alpha \beta}$ field one can determine the value of the ground state energy given by configurations of $H_{\mu \nu \alpha \beta}$. We are going to solve explicitly the classical equations of motion for the effective action (8). Then, the energy density associated with those solutions will be calculated.

Let us start with the equations of motion. Taking the variation of the action (8) with respect to the $C_{\nu \alpha \beta}(z)$ field one gets

$$
\partial^{\mu} H_{\mu \nu \alpha \beta}(z)=\theta \mathcal{X} \int_{\partial \Gamma} \delta^{(4)}(z-x) d x_{\nu} \wedge d x_{\alpha} \wedge d x_{\beta} .
$$

This equation can be solved exactly in four-dimensional space-time [27]. The solution is the sum of a particular solution of the inhomogeneous equation and a general solution of the corresponding homogeneous equation:

$$
H_{\mu \nu \alpha \beta}(z)=\theta \mathcal{X} \int \delta^{(4)}(z-x) d x_{\mu} \wedge d x_{\nu} \wedge d x_{\alpha} \wedge d x_{\beta}+b \varepsilon_{\mu \nu \alpha \beta} .
$$

The integration constant $b$, if nonzero, induces an additional $\mathrm{CP}$ violation beyond the existed $\theta$ angle. However, periodicity of the $\theta$ angle with respect to shifts by $2 \pi \times$ (integer) allows for some nonzero $b$ proportional to $2 \pi \mathbf{Z}$. As a result, the general solution to the equation of motion reads as follows:

\footnotetext{
$\S$ The action (\$) is not an effective action in the Wilsonian sense. It is rather related to the generating functional of one-particle-irreducible diagrams of the composite field in the large $N$ limit. The effective action in Eq. (8) is not to be quantized and loop diagrams of that action are not to be taken into account in calculating higher order Green's functions. The analogous effective action for the CP even part of the theory was constructed in Refs. [24], [25], (see also Ref. [26]).
} 


$$
H_{\mu \nu \alpha \beta}=-(\theta+2 \pi k) \mathcal{X} \varepsilon_{\mu \nu \alpha \beta} .
$$

Thus, the different vacua are labeled by the integer $k$ and the order parameter for these vacua in the large $N$ limit can be written as:

$$
\langle F \tilde{F}\rangle_{k}=(\theta+2 \pi k) \mathcal{X} .
$$

As a next step let us compute the vacuum energy associated with the solution given in Eq. (11). The density of the energy-momentum tensor for the action (8) takes the form

$$
\Theta_{\mu \nu}=-\frac{1}{3 ! \mathcal{X}}\left(H_{\mu \alpha \beta \tau} H_{\nu}{ }^{\alpha \beta \tau}-\frac{1}{8} g_{\mu \nu} H_{\rho \alpha \beta \tau}^{2}\right) .
$$

Using the expression (11) one calculates the corresponding energy density网 $\mathcal{E}_{k}$

$$
\mathcal{E}_{k}(\theta)=\frac{1}{2}(\theta+2 \pi k)^{2} \mathcal{X} .
$$

Since the $H_{\mu \nu \alpha \beta}$ field does not propagate dynamical degrees of freedom the expression above is the total energy density of the system given by the action (8)

Before we go further let us stop here to discuss some of the consequences of Eq. (14). First of all, let us notice that the result (14), as well as Eq. (11), is only valid in the limit of infinite $N$. In the next section we will calculate subleading order corrections to Eqs. (10,114) and argue that these expressions can also be used as a good approximation for large but finite $N$. The constant $C$ emerging in (1D) is related to the topological susceptibility as follows:

$$
\left.\mathcal{X}\right|_{N \rightarrow \infty}=2 C .
$$

Thus, the vacuum energy (1, 14) is defined by vacuum fluctuations of the topological charge measured by $\mathcal{X}$.

The crucial feature of (14) is that it defines an infinite number of vacua. The true vacuum is obtained by minimizing (14) with respect to $k$ as in (1):

$$
\mathcal{E}_{0}(\theta)=\frac{1}{2} \mathcal{X} \min _{k}(\theta+2 \pi k)^{2} .
$$

This expression is periodic with respect to shifts of $\theta$ by $2 \pi \mathbf{Z}$ and is also a smooth function of $\theta$ except for $\theta=\pi$ [12] (see also discussions below). Thus, there are an infinite number

\footnotetext{
** Notice that the total YM energy density should contain some negative constant related to the nonzero value of the gluon condensate [19]. This constant is subtracted from the expression for the energy discussed in this work. The energy density (14) is normalized as $\mathcal{E}_{0}(\theta=0)=0$, and for $k=0$ was discussed in [29].

${ }^{\dagger}$ One might wonder whether the same result is obtained if one treats $\theta$ not as a constant multiplying $Q$ in the Lagrangian, but as the phase that the states acquire under a topologically non-trivial gauge transformations. In this case the arbitrary integration constant in Eq. (10) has to be chosen in such a way which would guarantee a proper $\theta$ dependence of the VEV of the topological charge density. This would leave the results of our discussion without modifications.
} 
of the false vacua in the theory [12. The fate of these states will be discussed in section 4 .

\section{Large $N$ QCD calculation}

In this section we consider full QCD with three quark flavors. We are going to write down a low-energy effective Lagrangian for this case and then gradually decouple quarks by taking the quark masses to infinity. The resulting effective Lagrangian should be giving the energy density for pure Yang-Mills theory.

In the large $N$ expansion the effective Lagrangian of QCD with three flavors takes the form 30 32, 13]:

$$
\begin{gathered}
\mathcal{L}\left(U, U^{*}, Q\right)=\mathcal{L}_{0}\left(U, U^{*}\right)+\frac{1}{2} i Q(x) \operatorname{Tr}\left(\ln U-\ln U^{*}\right)+ \\
\frac{1}{2 \mathcal{X}} Q^{2}(x)+\theta Q(x)+\frac{B}{2 \sqrt{2}} \operatorname{Tr}\left(M U+M^{*} U^{*}\right)+\ldots,
\end{gathered}
$$

where $U$ denotes the flavor group matrix of pseudoscalar mesons, $\mathcal{L}_{0}$ denotes the part of the Lagrangian which contains the meson fields only [30 32,13], $B$ is some constant related to the quark condensate, and $M$ stands for the meson mass matrix (for recent discussions of the effective chiral Lagrangian approach see Ref. [34]). Higher order terms in (15) are suppressed by quadratic and higher powers of $1 / N$. In order to study vacuum properties, we concentrate on the low-momentum approximation. The Lagrangian presented above can be used to solve the $U(1)$ problem 17, 18. Indeed, the field $Q$ enters the Lagrangian in a quadratic approximation and can be integrated out. As a result, the flavor singlet meson, the $\eta^{\prime}$, gets an additional contribution into its mass term. This leads to the Witten-Veneziano relation and the solution of the $U(1)$ problem without instantons [17, 18]. In the present case we would like to follow an opposite way. Namely, we are going to make quarks very heavy and integrate them out keeping the field $Q$ in the Lagrangian. In the limit $m_{q} \rightarrow \infty$ one finds that $M \rightarrow \infty$. Thus, the low-energy effective Lagrangian which is left after the mesons are integrated out will take the form:

$$
\mathcal{L}_{\text {eff }}(Q)=\frac{1}{2 \mathcal{X}} Q^{2}(x)+\theta Q(x)+\mathcal{O}\left(\frac{\Lambda_{\mathrm{QCD}}^{2}}{M^{2}}, \frac{\partial^{2} Q^{2}}{\Lambda_{\mathrm{QCD}}^{6}}, \frac{1}{N^{2}}\right)
$$

Rewriting the field $Q$ in terms of the "massless" tensor $C_{\alpha \beta \gamma}$ as in the previous section, one finds that the expression (16) is nothing but the Lagrangian presented in (8). Thus, the higher order terms neglected in (8) which could contribute to the vacuum energy at zero momenta would correspond to higher corrections in $1 / N$. In fact, the subleading corrections to the effective Lagrangian (15) can also be found [33]. These terms are proportional (with the corresponding dimensionful coefficients) to the following expressions:

$$
\frac{\text { const. }}{N^{2}} Q^{2} \operatorname{Tr}\left(\partial_{\mu} U \partial_{\mu} U^{*}\right), \quad \frac{\text { const. }}{N^{2}} Q^{4}
$$

The terms listed above are suppressed in the effective Lagrangian by the factor $1 / N^{2}$. As a next step, we can include the terms (17) into the full effective Lagrangian and then integrate the heavy meson fields out. The net result of this procedure is that the terms proportional to $Q^{4}$ appear in the effective Lagrangian for pure YM theory. This, in its turn, modifies the 
equation of motion for the single component of $H_{\mu \nu \alpha \beta}$ considered in the previous section. Performing the calculation of the vacuum energy in the same manner as in section 2 we find the following result for the energy density:

$$
\mathcal{E}_{k}(\theta)=\frac{1}{2} \mathcal{X}(\theta+2 \pi k)^{2}+\frac{\text { const. }}{N^{2}} \mathcal{X}(\theta+2 \pi k)^{4}+\mathcal{O}\left(\frac{1}{N^{3}}\right)
$$

In this expression the arbitrary constant emerges as a result of integration of the equation of motion Notice that the topological susceptibility in the expression above is also defined in the corresponding order in the large $N$ expansion: $\mathcal{X}=2 C+\mathcal{X}_{1} / N+\mathcal{X}_{2} / N^{2}$. Thus, the expressions (14,18) could in principle give a reasonable approximation for big enough but otherwise finite $N$. The true vacuum energy density, $\mathcal{E}_{0}(\theta)$, can be obtained by minimizing the expression (18) with respect to $k$ as in (11). Then, $\mathcal{E}_{0}(\theta)$ satisfies the relation $\left.\partial_{\theta}^{2} \mathcal{E}_{0}(\theta)\right|_{\theta=0}=\mathcal{X}$, no matter what is the value of the arbitrary constant in (18).

\section{Dynamics of the false vacua}

In this section we will discuss the dynamics of the false vacua present in the theory. In accordance with (14, 18) there are an infinite number of vacua for any given value of the theta angle [12]. Clearly, not all of these are degenerate. The true vacuum state is defined by minimizing the expressions (14,18) with respect to $k$. All the other states are false vacua with greater values of the energy density. There is a potential barrier that separates a given false vacuum state from the true one. Thus, a false vacuum can in general decay into the true state through the process of bubble nucleation 35] 35. The decay rates for these vacua were evaluated recently in Ref. [36]. In this section we analyze the fate of the false vacua for different realizations of the initial conditions in which the system is placed. For the sake of simplicity we will be discussing transitions between the vacuum states labeled by $k^{\prime}$ and $k$ for different values of these integers. The first two cases considered in this section were studied in Refs. [12] and [36], we include them here for completeness.

\subsection{The false vacua with $k^{\prime} \sim 1$}

In this subsection we consider the system which in its initial state exists in a false vacuum with $k^{\prime}$ of order $\sim 1$. Let us start with the case when $N$ is a large but finite number so that the formula (14) (or (18)) is still a good approximation. Since there exists the true vacuum state with less energy, the false vacuum can "decay" into the true one via the bubble nucleation process. That is to say, there is a finite probability to form a bubble with the true vacuum state inside. The shell of the bubble is a domain wall which separates the false

\footnotetext{
${ }_{\ddagger}^{\ddagger}$ It is not clear, however, what is the numerical value of this constant, and whether in fact it is nonzero.

$\S \S$ This decay can go through the Euclidean "bounce" solution [37]. Though the existence of the bounce for this case is not easy to understand within the field theory context, nevertheless, one could be motivated by the brane construction where this object appears as a sixbrane bubble wrapped on a certain space [12].
} 
state from the true one. The dynamical question we discuss here is whether it is favorable energetically to create and expand such a bubble. Let us study the energy balance for the case at hand. While creating the shell of the bubble one looses the amount of energy equal to the surface area of the bubble multiplied by the tension of the shell. On the other hand, the true vacuum state is created inside the bubble, thus, one gains the amount of energy equal to the difference between the energies of the false and true states. The energy balance between these two effects defines whether the bubble can be formed, and, whether the whole false vacuum can transform into the true one by expanding this bubble to infinity. Let us start with the volume energy density. The amount of the energy density which is gained by creating the bubble is

$$
\Delta \mathcal{E}=\frac{1}{2} \mathcal{X}\left[(\theta+2 \pi)^{2}-\theta^{2}\right]=2 \pi \mathcal{X}(\theta+\pi) .
$$

Thus, $\Delta \mathcal{E}$ scales as $\sim 1$ in the large $N$ limit as long as the volume of the bubble is finite. Let us now turn to the surface energy which is lost. This energy is defined as:

$$
E_{s}=T_{D} \times(\text { surface area }) .
$$

The tension of the wall between the adjacent vacua, $T_{D}$, should scale as $T_{D} \sim N$ in the large $N$ limit [12]. Hence, the surface energy will also scale as $\sim N$. Thus, the process of creation of a finite volume bubble in the large $N$ limit is not energetically favorable. Indeed, the amount of energy which is lost while creating the shell is bigger than the amount which is gained. In terms of the false vacuum decay width this means that the width of this process is suppressed in the large $N$ limit [36]:

$$
\frac{\Gamma}{\text { Volume }} \propto \exp \left(-a N^{4}\right)
$$

where $a$ stands for some positive constant 36]. Thus, one concludes that in the limit $N \rightarrow \infty$ the false vacua with $k^{\prime} \sim 1$ are stable [12, 36].

\subsection{The false vacua with $k^{\prime} \sim N$}

Here we study the fate of the false vacua with $k^{\prime} \sim N$. We discuss a possibility that these vacua can decay into a state $k$ with $k^{\prime}-k \sim N$ and $k^{\prime}+k \sim N$. As in the previous subsection, we are going to study the energy balance for the bubble nucleation process. The amount of the volume energy density which is gained by creating such a bubble in the large $N$ limit scales as follows:

$$
\Delta \mathcal{E} \propto \mathcal{X} N^{2}
$$

Thus, the volume energy which is gained increases as $\sim N^{2}$. Let us now turn to the surface energy which is lost while nucleating a bubble. This is defined as $E_{s}^{\prime}=T_{D}^{\prime} \times($ surface area), where $T_{D}^{\prime}$ denotes the tension of the domain wall interpolating between the vacua labeled by

\footnotetext{
${ }^{* * *}$ In this subsection we assume that $\theta \neq \pm \pi$. The case $\theta=\pi$ will be considered below.
} 
$k^{\prime}$ and $k$. Since $k^{\prime}-k \sim N$ these vacua are not neighboring ones. Thus, in general, there is no reason to expect that the tension of the wall interpolating between these vacua scales as $\sim N . T_{D}^{\prime}$ might scale as $\sim N^{2}$ at most (as the energy of a generic configuration in a model with $N^{2}$ degrees of freedom). However, even in the case when $T_{D}^{\prime} \sim N^{2}$ the volume energy which is gained is at least of the same order as the surface energy which is lost. Hence, it is energetically favorable to increase the radius of such a bubble (since the volume energy scales as a cubic power of the radius while the surface energy scales only as a quadratic power of the effective size). Thus, the bubble nucleation process will not be suppressed and the false vacua with $k^{\prime} \sim N$ will eventually decay into the true ground state. Note, that the state $k^{\prime}=N$ can as well decay into the neighboring vacuum $k=N-1$ which subsequently is allowed to turn into the ground state.

\subsection{Parallel domain walls}

In this subsection we consider the special case when all the vacua are present simultaneously in the initial state of the model. This can be achieved, for instance, by placing in space an infinite number of parallel domain walls separating different vacua from each other. It is rather convenient to picture these walls as parallel planes. Each vacuum state is sandwiched between the corresponding two domain walls (two planes) separating this state from the neighboring vacua. Each domain is labeled by $k$ and in accordance with (14,18) is characterized by the corresponding value of the vacuum energy. Furthermore, the order parameter $\langle F \tilde{F}\rangle$ takes different values in these vacua in accordance with (12). Let us turn to the true vacuum state. For simplicity we assume that this state is given by $k=0$ (which corresponds to $|\theta|$ being less than $\pi$ ). The corresponding vacuum energy is the lowest one. Consider the two states which are adjacent to the true vacuum. These states have the energy density bigger than that of the true vacuum. Thus, there is a constant pressure acting on the domain walls separating the true vacuum from the adjacent false ones. This pressure will tend to expand the domain of the true vacuum. In fact, for large but finite $N$, the pressure will indeed expand the spatial region of the true vacuum by moving apart the centers of the domain walls sandwiching this state. The very same effect will be happening between any two adjacent vacua. Indeed, let us calculate the jump of the energy density between the two vacua labeled by $k^{\prime}$ and $k$ :

$$
\Delta \mathcal{E}_{k^{\prime} k}=2 \pi \mathcal{X}\left(k^{\prime}-k\right)\left(\theta+\pi\left(k^{\prime}+k\right)\right) .
$$

As far as $N$ is large but finite, the walls will start to accelerate. Farther the wall is located from the true vacuum (i.e. larger the sum $k^{\prime}+k$ ), bigger the initial acceleration of the wall is going to be; i.e., the walls will start to move apart from each other with the following initial acceleration:

$$
a_{k^{\prime} k} \propto \Lambda_{\mathrm{YM}} \frac{\left(k^{\prime}-k\right)\left[\theta+\pi\left(k^{\prime}+k\right)\right]}{N} .
$$

For finite $N$ all the walls will be moving to spatial infinity and the whole space will eventually be filled with the true vacuum state. On the other hand, when $N \rightarrow \infty$ the picture is a bit different. There are a number of interesting cases to consider:

First of all let us set $k^{\prime}-k=1$ and $k^{\prime}, k \sim 1$. Then, in the limit $N \rightarrow \infty$ the acceleration $a_{k^{\prime} k} \rightarrow 0$. Thus, the neighboring walls stand still if they had no initial velocity. The physical 
reason of this behavior is as follows: Though there is a constant pressure of order $\sim 1$ acting on the wall, nevertheless, the wall cannot be moved because the mass per unit surface area of the wall tends to infinity in the limit $N \rightarrow \infty$.

The second interesting case would be when the constant pressure produced by the energy jump between some neighboring vacua is of order $\sim N$. In this case it will be possible to accelerate these walls up to the speed of light and send them to spatial infinity. Indeed, if $k^{\prime}-k=1$ but $k^{\prime}+k \sim N$, then the wall between these two vacua starts moving with a non-vanishing acceleration which scales as follows:

$$
a_{k^{\prime} k} \propto \Lambda_{\mathrm{YM}} \frac{\pi\left(k^{\prime}+k\right)}{N} \sim \mathcal{O}(1)
$$

Thus, these walls will eventually be approaching spatial infinity with a speed of light even in the limit of infinite $N$.

In addition to the effects emphasized above there might also be decays of the false vacua happening in each particular domain. As we discussed in the previous subsections, for large but finite $N$ all the false vacua will be nucleating bubbles with energetically favorable phases inside and expanding these bubbles to infinity. Thus, for large but finite $N$, there are two effects which eliminate the false vacua: The moving walls are sweeping these states to infinity, and, in addition, these vacua are decaying via bubble nucleation processes.

What happens for an infinite $N$ ? As we learned above there are an infinite number of domains which will stay stable in that limit and the corresponding false vacua would not decay because of the exponential suppression. Thus, there are an infinite number of inequivalent spatial regions which are separated by domain walls. Consider one of the regions sandwiched between two domain walls. The three-form field $C_{\mu \nu \alpha}$ will couple to the walls and the large $N$ effective action for this case will look as follows:

$$
\tilde{S}=S_{\mathrm{eff}}+\sum_{i=k, k+1} \mu_{i} \int_{\mathcal{W}_{i}} C_{\mu \nu \alpha} d x^{\mu} \wedge d x^{\nu} \wedge d x^{\alpha},
$$

where $S_{\text {eff }}$ is defined in (8), $\mu_{i}$ stands for the coupling of the three-form potential to a corresponding domain wall; $\mathcal{W}_{i}$ denotes the worldvolume of the wall. In this case the domain wall can be regarded as a source of the corresponding three-form potential. This is reminiscent to what happens in the large $N$ supersymmetric YM model [38].

\subsection{Domain walls at $\theta=\pi$}

If $\theta=\pi$, the initial classical Lagrangian is CP invariant. Indeed, under CP transformations $\theta=\pi$ goes into $-\pi$. Since $\pi$ and $-\pi$ angles are equivalent, CP is a symmetry of the Lagrangian. However, in accordance with (12), this symmetry is spontaneously broken by the vacuum of the theory. Thus, one finds the following two degenerate true vacua:

$$
\mathcal{E}_{k=0}=\mathcal{E}_{k=-1}=\frac{1}{2} \mathcal{X} \pi^{2}
$$

\footnotetext{
${ }^{\dagger \dagger}$ I am grateful to Gia Dvali who pointed this out to me.
} 
These two vacua are labeled by the order parameter (12). In the $k=0$ state $\langle F \tilde{F}\rangle=\pi \mathcal{X}$ and in the $k=-1$ state $\langle F \tilde{F}\rangle=-\pi \mathcal{X}$. As a result of the spontaneous breaking of a discrete symmetry there should be a domain wall separating these two vacua. Let us consider the case discussed in the previous section. Namely, let us choose the initial condition of the system as a state where all the possible vacua are simultaneously realized in space. That is, there are an infinite number of domain walls (parallel planes) dividing space into an infinite number of domains with different values of the vacuum energy density labeled by $k$. As we mentioned above, only two of these domains have equal minimal energy density given in (25). The domain wall separating these two vacua, as we will see below, is somewhat special. In accordance with the discussions in the previous subsection, for large but finite $N$, all the walls merging with the false vacua will tend to rush to spatial infinity. The final stable state of the model can be described as a space separated into two parts by a single domain wall. To the left (right) of the wall one discovers the phase with $k=-1$ with the corresponding order parameter $\langle F \tilde{F}\rangle=-\pi \mathcal{X}$, and, to the right (left) of the wall one finds the state with $k=0$ and $\langle F \tilde{F}\rangle=\pi \mathcal{X}$. Vacuum energies of these two states are degenerate.

In the case of infinite $N$ the picture is slightly different. As elucidated in the previous subsection, there will be an infinite number of stable vacua. The domain wall separating the two true vacua can be regarded in this case as the fixed plane under $\mathbf{Z}_{2}$ transformations of the coordinate transverse to the plane. The three-form field $C_{\mu \nu \alpha}$ will be able to couple to this wall in a manner discussed in the previous subsection. The picture, being interpreted in the string theory approach, looks much like the one with an orientifold plane separating mirror $D$-branes on its both sides [39].

\section{A Comment on String Theory vs. Field Theory Approach}

We found in section 2 that the theta dependent vacuum energy (1) is related to the correlator (3) measuring the vacuum fluctuations of the topological charge. The question which arises here is whether this can be seen from the original string theory computation [12]. We are going to discuss below how the string theory calculation indicates that the vacuum energy (1) should indeed be related to the vacuum fluctuations of the topological charge. In fact, we argue that this is related to the instantons carrying D0-brane charge in the Type IIA fourbrane construction of the four-dimensional YM model.

To begin with let us recall how the theta dependent vacuum energy appears in the brane construction of the four-dimensional YM model [12]. One starts with Type IIA superstring theory on $\mathcal{M} \equiv \mathbf{R}^{4} \times \mathbf{S}^{1} \times \mathbf{R}^{5}$, with $N$ coincident $D$ 4-branes [5]. The $D$ 4-brane worldvolume is assumed to be $\mathbf{R}^{4} \times \mathbf{S}^{1}$ and the fermion boundary conditions on $\mathbf{S}^{1}$ are chosen in such a way that the low-energy theory on the worldvolume is pure non-supersymmetric $U(N)$ YM theory [5]. In the dual description, the large $N$ limit of the $S U(N)$ part of this theory can be studied by string theory on a certain background [2 [5]. It was shown in Ref. [12] that the theta dependent vacuum energy (1) arises in the dual string description due to the $U(1)$ gauge field $B_{M}, M=1, . ., 5$. To find out what this corresponds to in the original gauge theory language recall that this $U(1)$ field is nothing but the Ramond-Ramond (RR) oneform of Type IIA theory. Furthermore, once the gauge theory is realized in the Type IIA fourbrane construction, the Wess-Zumino-Witten (WZW) term present in the worldvolume effective action defines the correspondence between the gauge theory operators on one side and the string theory Ramond-Ramond fields on the other side. In the case at hand the 
worldvolume WZW term looks as follows:

$$
S_{\mathrm{WZW}}=\frac{1}{8 \pi^{2}} \int_{\mathcal{V}} B \wedge \operatorname{Tr} F \wedge F
$$

where $\mathcal{V}$ denotes the worldvolume of a wrapped fourbrane, $\mathcal{V} \equiv \mathbf{R}^{4} \times \mathbf{S}^{1}$. In accordance with the general principles of the large $N$ AdS/CFT correspondence [2] the classical action for the RR one-form on the string theory side defines the YM correlation functions of the composite operator $F \tilde{F}$ (since this is the operator which couples to the corresponding RR field in (26)). Moreover, the two-point correlator of the topological charge density $F \tilde{F}$, which coincides with $\mathcal{X}$ in (3) up to some contact term [17], was recently calculated using the AdS/CFT correspondence and the supergravity analyses for the RR one-form $B_{M} 40$. Thus, it is not surprising that the theta dependent vacuum energy which is defined by the $\mathrm{RR}$ one-form in the string theory calculation is related to the nonzero value of the topological susceptibility (3) in the gauge theory studies. The physical reason for this correspondence, as we have mentioned above, is the special property of the gauge theory instantons in the fourbrane construction. Indeed, in accordance with (26) the RR one-form couples to the topological charge density $F \tilde{F}$, on the other hand the RR one-form couples by definition to D0-branes. Thus, the gauge theory instantons in this case carry zerobrane charge. This is the physical reason for the correspondence discussed above.

\section{Conclusions}

Let us summarize briefly the results of this work. The expression for the theta dependent vacuum energy derived in Ref. [12] in a string theory calculation was obtained in large $N$ pure YM theory. This energy is defined by vacuum fluctuations of the topological charge. The field-theoretic approach allows one to clarify the puzzle why the theta dependent vacuum energy is of order $\sim 1$ in the large $N$ limit. The field which saturates the expression for the energy has very specific properties, it produces the background energy density but does not propagate any dynamical degrees of freedom in the large $N$ limit. Within the field theory framework subleading corrections to the large $N$ result (11) were calculated (see Eq. (18)).

Given the expression for the theta dependent vacuum energy we studied the issue of stability of the false vacua for different realization of the initial conditions of the system. The large but finite $N$ and infinite $N$ cases were considered. The structure of the vacuum state in these two cases is rather different.

The string theory calculation provides important clues on what the theta dependent vacuum energy should be related to within the field theory approach. We discussed this issue arguing that the reconciliation of the field theory result with the original string theory

calculation is based on the fact that instantons carry D0-brane charge in the D4-brane construction of pure YM model.

\section{Acknowledgments}

The author is grateful to Gia Dvali for reading the manuscript and for useful discussions and suggestions. The work was supported in part by the grant NSF PHY-94-23002. 


\section{REFERENCES}

[1] For a recent review, see:

A. Giveon, D. Kutasov, "Brane Dynamics and Gauge Theory", hep-th/9802067.

[2] J. Maldacena, "The Large $N$ limit of Superconformal Field Theories and Supergravity", Adv. Theor. Math. Phys. 2 (1998) 231; hep-th/9711200.

[3] S.S. Gubster, I.R. Klebanov, A.M. Polyakov, "Gauge Theory Correlators from Noncritical String Theory", Phys. Lett. B428 (1998) 105; hep-th/9802109.

[4] E. Witten, "Anti-de Sitter Space and Holography", Adv. Theor. Math. Phys. 2 (1998) $253 ;$ hep-th/9802150.

[5] E. Witten, "Anti-de Sitter Space, Thermal Phase Transitions, and Confinement in Gauge Theories", Adv. Theor. Math. Phys. 2 (1998) 505; hep-th/9803131.

[6] D. J. Gross, H. Ooguri, "Aspects of Large $N$ Gauge Theory Dynamics as Seen by String Theory", Phys. Rev. D58 (1998) 106002; hep-th/9805129.

[7] C. Csaki, H. Ooguri, Y. Oz, J. Terning, "Glueball Mass Spectrum from Supergravity", hep-th/9806021; R. de Mello Koch, A. Jevicki, M. Mihailescu, J.P. Nunes, "Evaluation of Glueball Masses from Supergravity", Phys. Rev. D58 (1998) 105009; hep-th/9806125; M. Zyskin, "A Note on the Glueball Mass Spectrum", Phys. Lett. B439 (1998) 373; hep-th/9806128;

[8] H. Ooguri, H. Robins, J. Tannenhauser, "Glueballs and their Kaluza-Klein Cousins", Phys. Lett. B437 (1998) 77.

[9] I.R. Klebanov, "From Threebranes to Large N Gauge Theories", hep-th/9901018.

[10] I.R. Klebanov, A.A. Tseytlin, "D-branes and Dual Gauge Theories in Type 0 Strings", hep-th/9811035.

[11] J. Minahan, "Glueball Mass Spectra and Other Issues for Supergravity Duals of QCD Models", hep-th/9811156.

[12] E. Witten, "Theta dependence in the Large $N$ Limit of Four-Dimensional Gauge Theories", Phys. Rev. Lett. 81 (1998) 2862; hep-th/9807109.

[13] E.Witten, "Large N Chiral Dynamics", Ann. of Phys. 128 (1980) 363.

[14] A.M. Belavin, A.M. Polyakov, A.S. Schwartz, Yu.S. Tyupkin, "Pseudoparticle Solutions of the Yang-Mills Equations", Phys. Lett. 59B (1975) 85.

[15] S. Coleman, "Aspects of Symmetry", Cambridge University Press, 1985.

[16] E.V. Shuryak, "The Role of Instantons in Quantum Chromodynamics. 1. Physical Vacuum", Nucl.Phys. B203 (1982) 93;

D.I. Diakonov, V.Y. Petrov, "Instanton Based Vacuum from Feynman Variational Principle", Nucl.Phys. B245 (1984) 259;

T. DeGrand, A. Hazenfratz, T.G. Kovacs, "Topological Structure in the $S U(2)$ Vacuum", Nucl. Phys. B505 (1997) 417; hep-lat/9705009.

[17] E. Witten, "Current Algebra Theorems for the U(1) "Goldstone Boson" ", Nucl. Phys. B156 (1979) 269.

[18] G. Veneziano, "U(1) Without Instantons", Nucl. Phys. B159 (1979) 213.

[19] M.A. Shifman, A.I. Vainshtein, V.I. Zakharov, "QCD and Resonance Physics. Theoretical Foundations", Nucl. Phys. B147 (1979) 385.

[20] G. Grunberg, "QCD Sum Rule Determination of the Topological Susceptibility", Phys. Rev D30 (1984) 1570. 
[21] B. Alles, M. D'Elia, A. Di Giacomo, "Topological Susceptibility at Zero and Finite T in SU(3) Yang-Mills Theory", Nucl. Phys. B494 (1997) 281; hep-lat/9605013.

[22] M. Lüscher, "The Secret Long Range Force in Quantum Field Theories With Instantons", Phys. Lett. 78B (1978) 465.

[23] J. Kogut, L. Susskind, "How to Solve the $\eta \rightarrow 3 \pi$ Problem by Seizing the Vacuum ", Phys. Rev. D11 (1975) 3594.

[24] J. Schechter, "Effective Lagrangian With Two Color Singlet Gluon Fields", Phys. Rev. D21 (1980) 3393.

[25] A.A. Migdal, M.A. Shifman, "Dilaton Effective Lagrangian in Gluodynamics", Phys. Lett. 114B (1982) 445.

[26] T. Fugleberg, I. Halperin, A. Zhitnitsky, "Domain Walls and Theta Dependence in QCD with an Effective Lagrangian Approach", hep-ph/9808469.

[27] A. Aurilia, "The Problem of Confinement: From Two to Four Dimensions", Phys. Lett. 81B (1979) 203.

[28] A. Aurilia, H. Nicolai, P.K. Townsend, "Hidden Constants: The $\theta$ Parameter of QCD and the Cosmological Constant of $N=8$ Supergravity", Nucl. Phys. B176(1980)509.

[29] G. Gabadadze, "Modeling the Glueball Spectrum by a Closed Bosonic Membrane", Phys. Rev. D58 (1998) 094015; hep-ph/9710402.

[30] P. Di Vechia, G. Veneziano, "Chiral Dynamics in the Large $N$ Limit", Nucl. Phys. B171 (1980) 253.

[31] R. Arnowitt, Pran Nath, "The $U(1)$ Problem: Current Algebra and the Theta Vacuum", Phys. Rev. D23 (1981) 473; "Effective Lagrangians with $U(1)$ Axial Anomaly. 1, 2", Nucl. Phys. B209 (1982) 234, 251;

[32] C. Rosenzweig, J. Schechter, C.G. Trahern, "Is the Effective Lagrangian for QCD a Sigma Model?", Phys. Rev. D21 (1980) 3388.

[33] P. Di Vecchia, F. Nicodemi, R. Pettorino, G. Veneziano, "Large N, Chiral Approach to Pseudoscalar Masses, Mixings and Decays", Nucl. Phys. B181 (1981) 318.

[34] A. Pich, "Chiral Perturbation Theory", Rept. Prog. Phys. 58 (1995) 563; hep$\mathrm{ph} / 9502366$.

[35] M. B. Voloshin, I. Yu. Kobzarev, L. B. Okun', "Bubbles in Metastable Vacuum", Yad. Fiz. 20 (1974) 1229 (Sov. J. Nucl. Phys. 20 (1975) 644);

S. Coleman, "The Fate of the False Vacuum. 1. Semiclassical Theory", Phys. Rev D15 (1977) 2929; Erratum-ibid. D16 (1977) 1248.

[36] M. Shifman, "Domain Walls and Decay Rate of the Excited Vacua in the Large $N$ Yang-Mills Theory", Phys. Rev D59 (1999) 021501; hep-th/9809184.

[37] S. Coleman, "The Fate of the False Vacuum. 1. Semiclassical Theory", Phys. Rev D15 (1977) 2929; Erratum-ibid. D16 (1977) 1248;

C. Callan, S. Coleman, "The Fate of the False Vacuum. 2. First Quantum Corrections", Phys. Rev. D16 (1977) 1762.

[38] G. Dvali, G. Gabadadze, Z. Kakushadze, "BPS Domain Walls in Large N Supersymmetric QCD", hep-th/9901032.

[39] J. Polchinski, S. Chaudhuri, C.V. Johnson, "Notes on D-branes", hep-th/9602052;

J. Polchinski, "TASI Lectures on D-branes", hep-th/9611050.

[40] A. Hashimoto, Y. Oz, "Aspects of QCD Dynamics from String Theory", hepth/9809106. 